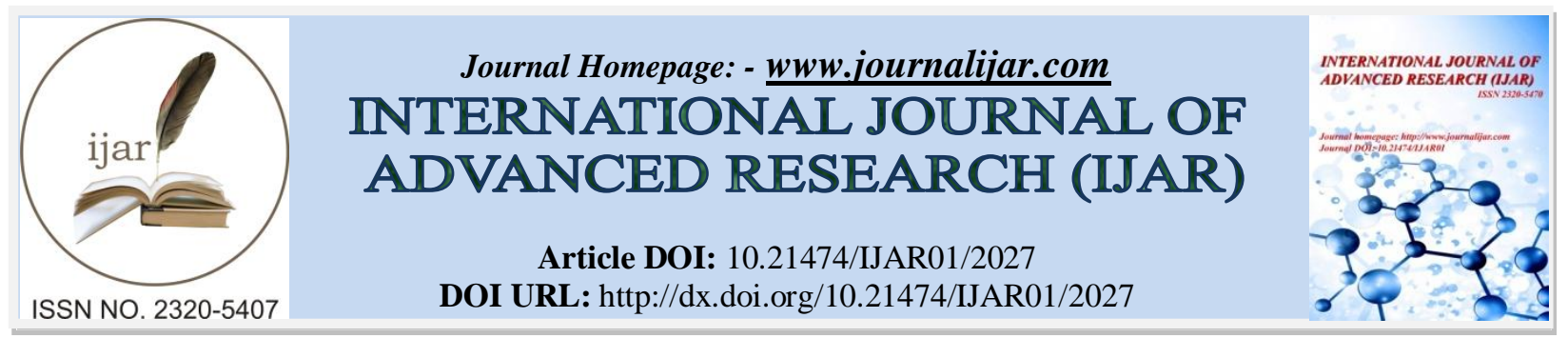

RESEARCH ARTICLE

\title{
SYNTHESIS, CHARACTERIZATION AND STUDY OF BIOLOGICAL ACTIVITIES SOME NEW CHALCONE DERIVATIVES CONTAINING DIPHENYL AMINE MOIETY USING RECYCLABLE CATALYST SYSTEM.
}

\author{
Manju Yadav*, Asha Lavania, Kiran Dasaray, Anita V. K. Anand, and Ajay Singh. \\ Department of chemistry - school of chemical science St. John's College, Agra - 282 002, India.
}

\section{Manuscript Info}

Manuscript History

Received: 25 September2016

Final Accepted: 9 October 2016

Published: October 2016

Key words:-

Chalcones, acetyl diphenyl amine, Aromatic aldehydes, Aqueous $\mathrm{NaOH}$, PEG 400, Antimicrobial activity.

\section{Abstract}

In an effort to develop a series of substituted chalcone derivatives have been synthesized by claisen - Schmidt condensation. In the present study some new chalcones have been synthesized by the reaction of acetyl diphenyl amine with different aromatic aldehydes in the presence of aqueous solution of sodium hydroxides ethanol and PEG 400 used as a catalyst at room temperature. The structure of various synthesized compounds are assigned on the basis of elemental analysis, IR, NMR and Mass spectral data, the synthesized compounds were screened for antimicrobial activity.

Copy Right, IJAR, 2016,. All rights reserved.

\section{Introduction:-}

Heterocyclic compounds are widely distributed in nature and occupy a prominent place in medicinal, pharmaceuticals and as drug intermediate.

Chalcones structurally represent open chain flavonoid in which the two aromatic rings are joined by a three - carbon $\alpha, \beta$ - unsaturated carbonyl system (1,3-diphenyl - 2 - propen - 1- one)

Chalcones are possess different types of biological activity ${ }^{1}$ such as antimicrobial ${ }^{2}$ anti-inflammatory ${ }^{3}$, analgesic ${ }^{4}$, antipletlet $^{5}$, antiulcerative ${ }^{6}$, antifungal ${ }^{7}$, anti-malarial ${ }^{8}$, anti plasmodial, anti cancer ${ }^{9}$ anti viral ${ }^{10}$, antileishmanial ${ }^{11}$, antioxidant, anti-tubercular activities ${ }^{12-15}$.

Chalcones and its derivatives are primary synthesized in the laboratory using claisen-schmidt reaction, in which acetophenon or its derivatives is reacted with benzaldehyde or its derivatives using strong base. Such as $\mathrm{NaOH}$ or $\mathrm{KOH}$ or these day PEG is used as a catalyst, shown in following reaction.

\section{Materials and methods:-}

Acetyl-diphenyl amine and all aromatic aldehyde were purchased from sigma Aldrich. PEG (400) used was of Thomas backer. Ethanol and other chemicals of AR grade were used as received.

\section{Experimental:-}

Synthesis of chalcone is a single step method. A mixture of acetyl-diphenyl amine (0.01) (mol) and appropriate aldehyde (R) or 0.01 (mol) was strirred in ethanol and then aqueous solution of $\mathrm{NaOH} 40 \%$, 10ml) was added to it

\section{Corresponding Author:- Manju Yadav.}

Address:- Department of chemistry - school of chemical science St. John's College, Agra - 282002 , India. 
The sodium salt of chalcone separated was decomposed by ice-cold $\mathrm{HCl}(30 \%)$ chalcone was filtered, washed with water $(50 \mathrm{ml})$ dried and recrystalized from absolute ethanol.
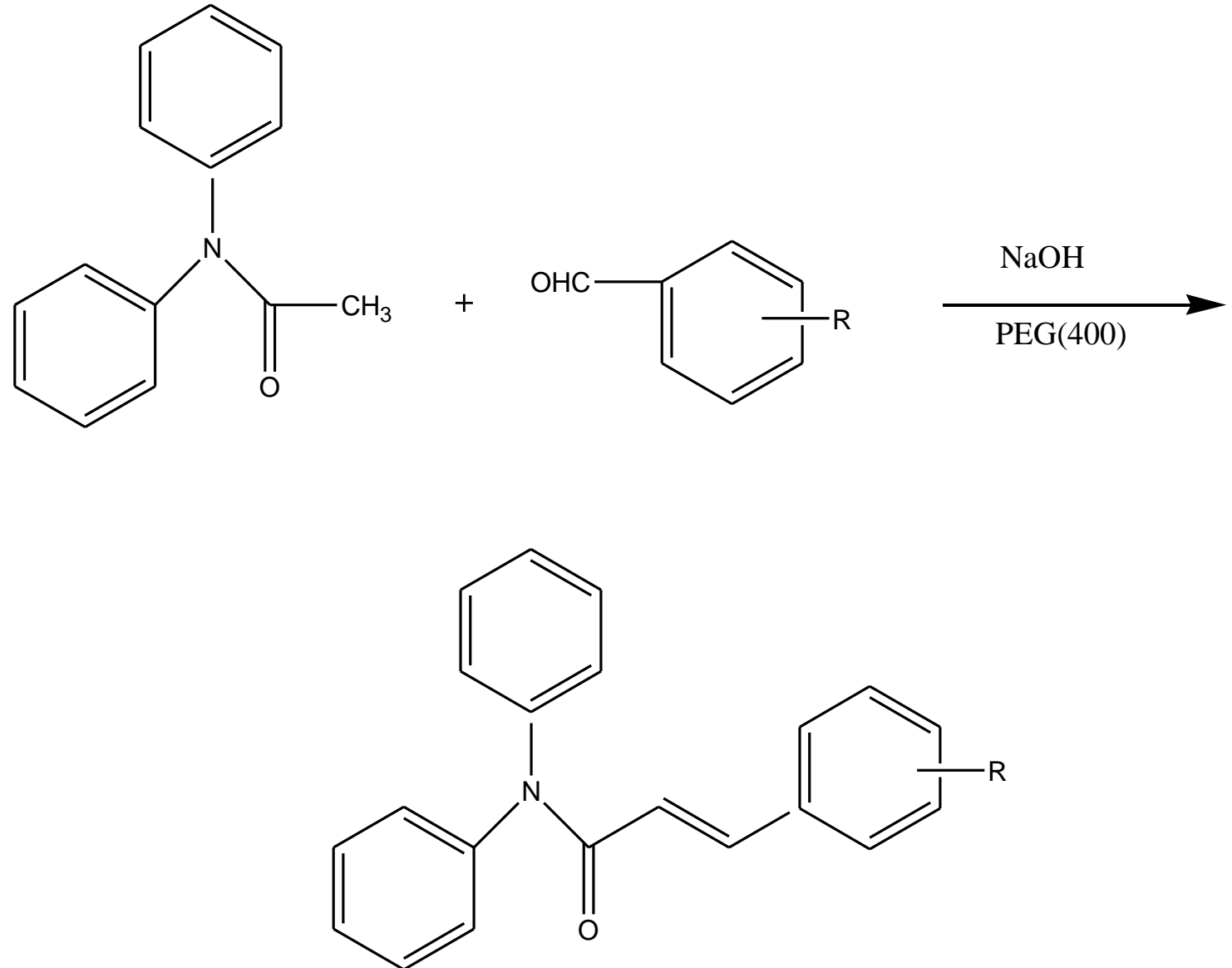

Where $\mathrm{R}=$ Substituted Aldehyde

(Scheme - 1 Synthesis of some new chalcones of acetyl diphenyl amine)

The yield of the synthesized compounds was found to be significant in the presence of PEG(400) catalyst. Elemental analysis confirmed percentage of carbon, hydrogen and oxygen to be similar to the calculated values.

\section{Physical measurements and analytical data:-}

The melting point were determined in open capillaires on electro thermal apparatus and are uncorrected the purity of all compounds were checked by thin layer chromatography using protected silica $60 \mathrm{~F}-254$ plate using hexane, ethylacetate, chloroform as mobile phase.

Table:- Calcone Obtained by the condensation of $\mathrm{N}$-acetyl diphenylamine with various substituted aldehydes

\begin{tabular}{|c|c|c|c|c|c|c|c|c|c|c|c|c|}
\hline \multirow{2}{*}{$\begin{array}{l}\text { S. } \\
\text { No. }\end{array}$} & \multirow{2}{*}{$\begin{array}{l}\text { Substituted } \\
\text { (R) }\end{array}$} & \multirow{2}{*}{$\begin{array}{l}\text { Molecular } \\
\text { Formula }\end{array}$} & \multirow{2}{*}{$\begin{array}{c}\text { M.P. } \\
{ }^{\circ} \mathrm{C}\end{array}$} & \multirow{2}{*}{$\begin{array}{c}\% \\
\text { Yield }\end{array}$} & \multicolumn{2}{|c|}{ Carbon (\%) } & \multicolumn{2}{|c|}{ Hydrogen (\%) } & \multicolumn{2}{|c|}{ Nitrogen (\%) } & \multicolumn{2}{|c|}{ Oxygen (\%) } \\
\hline & & & & & Found & Calcd. & Found & Calcd. & Found & Calcd. & Found & Calcd. \\
\hline 1. & Benzaldehyde & $\mathrm{C}_{21} \mathrm{H}_{17} \mathrm{NO}$ & 209 & 90 & 84.28 & 84.25 & 5.68 & 5.72 & 4.68 & 4.67 & 5.35 & 5.34 \\
\hline 2. & $\begin{array}{l}\text { 4-methoxy } \\
\text { Benz. }\end{array}$ & $\mathrm{C}_{22} \mathrm{H}_{19} \mathrm{NO}_{2}$ & 255 & 84 & 80.24 & 80.22 & 5.77 & 5.81 & 4.44 & 4.25 & 9.72 & 9.71 \\
\hline 3. & $\begin{array}{l}\text { 3-bromo } \\
\text { Benz. }\end{array}$ & $\mathrm{C}_{21} \mathrm{H}_{16} \mathrm{BrNO}$ & 281 & 85 & 66.66 & 66.68 & 4.23 & 4.26 & 3.70 & 3.70 & 4.23 & 4.23 \\
\hline 4. & $\begin{array}{l}\text { 3-methoxy 4- } \\
\text { hyd Benz. }\end{array}$ & $\mathrm{C}_{22} \mathrm{H}_{19} \mathrm{NO}_{3}$ & 367 & 80 & 76.52 & 76.50 & 5.50 & 5.54 & 4.05 & 4.06 & 13.91 & 13.90 \\
\hline 5. & $\begin{array}{l}\text { 2-chloro 6- } \\
\text { fluro Benz. }\end{array}$ & $\mathrm{C}_{21} \mathrm{H}_{15} \mathrm{ClFNO}$ & 265 & 81 & 71.79 & 71.70 & 4.27 & 4.30 & 3.98 & 3.98 & 4.55 & 4.55 \\
\hline
\end{tabular}


Microanalysis of carbon, hydrogen and oxygen of the compounds were carried on a Heraeus Carlo Erba 1108 elemental analyzer. IR spectra were recorded on perkin-elemer RX-1 infrared spectrometer in the range 4000-450 $\mathrm{cm}^{-1},{ }^{1} \mathrm{HNMR}, 13 \mathrm{C}-\mathrm{NMR}$ spectra of the compounds were recorded on a Bruker Advance $300 \mathrm{MHz}$. The ESI ${ }^{+}$mass spectra were recorded on a JEOL-Accu TOF JMS - 100LC mass spectrometer.

\section{Result and Discussion:-}

That data obtained of the synthesized compounds.

(diphenylamine-yl)-3-(phenyl)-prop-2 ene-1one-(a) shiny yellow crystal, yield-90\%, mp. $209^{\circ} \mathrm{C}$, Anal. Caltd for $\mathrm{C}_{21} \mathrm{H}_{17} \mathrm{NO}, \mathrm{C}=84.25, \mathrm{H}=5.73 \mathrm{~W}=4.68, \mathrm{O}=5.34(\%)$ found $\mathrm{C}=84.28 . \mathrm{H}=5.68, \mathrm{~N}=4.68, \mathrm{O}=5.35(\%), \mathrm{IR}\left(\mathrm{cm}^{-1}\right) 3423$ $(\mathrm{C}=0) 1657(\mathrm{C}=\mathrm{C}), 1402(\mathrm{C}-\mathrm{O}-\mathrm{C}),{ }^{1} \mathrm{HNMR}\left(400 \mathrm{MHz} \mathrm{CdCl}_{3}\right), \square($ inppm), 7.85 (d;2H, Ar-H), 7.38-7.35(d, 2H, Ar-H), 7.25 (d, 1HRCH) $7.15-7.06$ (m, 2H, S-H), ${ }^{13} \mathrm{CNMR}\left(100 \mathrm{MHz} ; \mathrm{CdCl}_{3}\right) \square$ (inppm) 168.89, 135.65, 134.01, 129.91, $129.53,120.41-41, \mathrm{MS} \mathrm{m} / \mathrm{z} 299\left(\mathrm{~m}^{+}\right)$.

(diphenylamine-yl)-3-(4-methoxyphenyl)prop-2-ene-1-one (b) light shiny brown, yield $84 \%, \mathrm{mp} 225^{\circ} \mathrm{C}$, and caltd for $\mathrm{C}_{22} \mathrm{H}_{19} \mathrm{NO}_{2} \mathrm{C}=80.22, \mathrm{H}=5.81, \mathrm{~N}=4.25, \mathrm{O}=9.71(\%)$ found $\mathrm{C}=80.24, \mathrm{H}=5.77, \mathrm{~N}=4.44, \mathrm{O}=9.72(\%), \quad \mathrm{IR}\left(\mathrm{cm}^{-1}\right)$ $1692(\mathrm{C}=0), 1562(\mathrm{C}=\mathrm{C}) 1272(\mathrm{C}-\mathrm{O}-\mathrm{C}),{ }^{1} \mathrm{HNMR}\left(400 \mathrm{MH}^{-} \mathrm{CdCl}_{3}\right) \square(\mathrm{inppm})=7.89,(\mathrm{~S}, 1 \mathrm{H}, \mathrm{CH}), 7.427 .37(\mathrm{~d} 2 \mathrm{H}$ Ar-H), 7.30 (S, 1H, CH), 7.20-1.13 (M, 2H, Ar-H), 13C-NMR (100 MHz CdCl 3 ) $\square$ (inppm) 172.40, 138.70, 135.02, 132.10, 130.80, 123.20 MS M/z $329\left(\mathrm{M}^{+}\right)$

(diphenyl amine-yl)-3-(3-bromo) Prop-2ene-1 one (c) : light yellow, yield, $85 \%$, M.P. $-281^{\circ} \mathrm{C}$ And Caltd for $\mathrm{C}_{21} \mathrm{H}_{16} \mathrm{BrNO}, \mathrm{C}=66.68, \mathrm{H}=4.23, \mathrm{Br}=21.13, \mathrm{~N}=3.70, \mathrm{O}=4.23(\%)$, IR $\left(\mathrm{cm}^{-1}\right), 1695(\mathrm{C}=\mathrm{O}) .1565(\mathrm{C}=\mathrm{C}), 1275(\mathrm{C}-\mathrm{O}-\mathrm{C})$ 1HNMR (400 MHz $\left.\mathrm{CdCl}_{3}\right) \square($ inppm) $=7.90(\mathrm{~S}, 1 \mathrm{H}, \mathrm{CH}), 7.45-7.38$ (d, 2H, Ar-H) 7.32 (S, 1H, CH) 7.22-7.15 (M, $\left.2 \mathrm{H}, \mathrm{Ar}-\mathrm{H}), 13 \mathrm{C}-\mathrm{NMR}(100 \mathrm{MHz} \mathrm{CdCl})_{3}\right) \square($ inppm $)=190.90,145.60,140.02,135.20,132.40,125.10, \mathrm{MS} \mathrm{M} / \mathrm{z} 378$ $\left(\mathrm{M}^{+}\right)$.

(diphenyl amine-yl)-3-(3-methoxy-4 Hydroxy Phenyl) Prop-2ene-1 one (d) : light cream, yield, 80\%, M.P. $267^{\circ} \mathrm{C}$ Anal Caltd for $\mathrm{C}_{22} \mathrm{H}_{19} \mathrm{NO}_{3}, \mathrm{C}=76.50, \mathrm{H}=5.54, \mathrm{~N}=4.06, \mathrm{O}=13.90(\%)$, found $-\mathrm{C}=76.52, \mathrm{H}=5.50, \mathrm{~N}=4.05$, $\mathrm{O}=13.91 \%$, IR $\left(\mathrm{cm}^{-1}\right), 1700(\mathrm{C}=\mathrm{O}) .1575(\mathrm{C}=\mathrm{C}), 1280(\mathrm{C}-\mathrm{O}-\mathrm{C})$ 1HNMR $(400 \mathrm{MHz} \mathrm{CdCl}) \square($ inppm $)=7.92(\mathrm{~S}, 1 \mathrm{H}$, $\mathrm{CH})$, 7.50-7.42 (d, 2H, Ar-H) 7.35 (S, 1H, CH) 7.32-7.19 (M, 2H, Ar-H), 13C-NMR (100 MHz CdCl 3 ) $\square($ inppm) $=$ $175.80,140.80,137.10,133.20,132.10,125.10, \mathrm{MS} \mathrm{M} / \mathrm{z} 345\left(\mathrm{M}^{+}\right)$.

(diphenyl amine-yl)-3-(2-chloro-6-fluro) Prop-2ene-1 one (e) : white, yield, $81 \%$, M.P. $-265^{\circ} \mathrm{C}$ Anal Caltd for $\mathrm{C}_{21} \mathrm{H}_{15} \mathrm{ClFNO}, \mathrm{C}=71.70, \mathrm{H}=4.30, \mathrm{Cl}=10.08, \mathrm{~F}=5.40, \mathrm{~N}=3.98, \mathrm{O}=4.55$, found $\mathrm{C}=71.79, \mathrm{H}=4.27, \mathrm{Cl}=10.11, \mathrm{~F}=5.41$, $\mathrm{N}=3.98, \mathrm{O}=4.55(\%), \mathrm{IR}\left(\mathrm{cm}^{-1}\right), 1720(\mathrm{C}=\mathrm{O}) .1590(\mathrm{C}=\mathrm{C}), 1295(\mathrm{C}-\mathrm{O}-\mathrm{C}) ; 1 \mathrm{HNMR}\left(400 \mathrm{MHz} \mathrm{CdCl}_{3}\right) \square($ inppm $)=$ 7.98 (S, 1H, CH), 7.55-7.45 (d, 2H, Ar-H) 7.37 (S, 1H, CH) 7.35-7.25 (M, 2H, Ar-H), 13C-NMR (100 MHz CdCl $)_{3}$ $\square($ inppm $)=177.90,142.70,138.20,134.1,133.20,127.20 \mathrm{MS} \mathrm{M} / \mathrm{z} 351\left(\mathrm{M}^{+}\right)$.

\section{Biological Activity:-}

The biological activity was performed by filter paper disc plate method.

\section{Antibacterial activity:-}

All human pathogenic bacteria viz Klebsiella $\left(\mathrm{gm}^{+\mathrm{ve}}\right)$, Escherichia coli, staphylococci $\left(\mathrm{gm}^{+\mathrm{ve}}\right)^{16}$ were purchased from Ajay Diagnostic and Research centre Jay Hospital, Agra India.

\section{Antifungal Activity:-}

The synthesized compounds were screened for their in vitro antifungal activity against Aspergillus niger, Aspergillus fumigatus, Candida albicans by measuring the zone of inhibition in $\mathrm{mm} .{ }^{17-18}$ 
Antibacterial \& Antifungal data of compounds

Zone of inhibition measured in $\mathrm{mm} \mathrm{MIC}=10 \square \mathrm{g} /$ disc .

\begin{tabular}{|c|c|c|c|c|c|c|}
\hline Comp. & \multicolumn{3}{|c|}{ Antifacterial } & \multicolumn{3}{c|}{ Antifungal } \\
\cline { 2 - 7 } & $\begin{array}{c}\text { Klebsiella } \\
\text { pneumoniae }\end{array}$ & $\begin{array}{c}\text { Escherichia } \\
\text { coli }\end{array}$ & $\begin{array}{c}\text { Staphylococcus } \\
\text { aureus }\end{array}$ & $\begin{array}{c}\text { Aspergillus } \\
\text { niger }\end{array}$ & $\begin{array}{c}\text { Asperigillus } \\
\text { fumigatus }\end{array}$ & $\begin{array}{c}\text { Candida } \\
\text { albicans }\end{array}$ \\
\hline $\mathrm{a}$ & 08 & 07 & 08 & 08 & 07 & 07 \\
\hline $\mathrm{b}$ & 07 & 06 & 06 & 07 & 08 & 06 \\
\hline $\mathrm{c}$ & 06 & 07 & 08 & 06 & 07 & 08 \\
\hline $\mathrm{d}$ & 08 & 06 & 09 & 08 & 09 & 07 \\
\hline $\mathrm{e}$ & 08 & 07 & 08 & 06 & 08 & 06 \\
\hline
\end{tabular}

\section{Application:-}

This is an environmentally benign procedure and reduces the total reaction time and gave excellent yield of chalcones.

\section{Conclusion:-}

The present work demonstrated the synthesis of chalcone in excellent yield in the presence of a reusable and environmentally benign reaction catalyst PEG(400), the structure of the synthesized compounds were confirmed from their respective, IR, 1HNMR, 13C NMR and Mass studies. From the Antimicrobial screening it was observed that all the compounds exhibited activity against all the organisms employed, the compound shows good antibacterial and antifungal activity.

\section{Acknowledgement:-}

We are thankful to department of Chemistry, School of Chemical Science, St. John's College, Agra for providing laboratory facilities and Central Drug Research Institute Lucknow for spectral data. We are also thankful to Dr. Ajay Singh, Ajay Diagnostic \& Research Centre (Jay Hospital, Agra) for the screening of Biological Activity.

\section{Reference:-}

1. Tenover, F.C., Medonald, L.C. Cuur. Opin, infect Dis 2005, 18, 300.

2. Y.R. Prasad; P.P.Kumar, C.A. Deepti; M.V. ramana, E. Journal of Chemistry; 2006 3(13), 236-241.

3. H. K. Hsieh, L.T. Tsao, J.P. Wang, J. Pharm Pharmacol 2000, 52, 163.

4. G.S. Viana, M.A. Banderia, F. Mattos, J. Phytomedicine 2003, 10, 189.

5. L. M. Zhao, H.S., Jin, L.P. Sun, H.R. Piao, Z.S. Quan, Bioorg. Med Chem, Lett. 2005, 15, 5027.

6. S. Mukarami, M. Muramastu, H. Aihara, S. Otomo. Biochem., Pharmacol, 1991, 42, 1447.

7. A., Mukherjee, N., Chaudhary, A. N., Experienta, 1974, 30, 1022.

8. M. Liu, P. Wilairat, L.M. Go, J. Med. Chem. 2001, 44, 4443.

9. E. Francesco, G. Salvatore, M. Luigi, C. Massimo,Phytochem, 2007, 68, 939.

10. J. C. Onyilagna, B. Malhotra, M. Elder, G.H.N. Towers, Can. J. Plant Pathol, 1997, 19, 133.

11. S.F. Nielsen, M. Chen. T.G. Theander, A. Kharazmi, S.B. Christensen, Bioorg. Med. Chem., 2004, 12, 883.

12. S. K. Kumar, E. Hager, C. Pethit, H. Gurulingappa; N.E. Davidson; S.R. Bioorg. Med. Chem, 2004, 12, 883.

13. J. Tasuzaki; K.F. Bastow; K. Nakagawa-Goto, S, Nakura, H. Itokawa, K.H. Lee, J. Netprod, 2006, 69(10), 1445-1445.

14. J. M. Yun, M.H. Kweon; H. Kwon; J.K. Hwanj; H. Mukhtar, Carcinogensis, 2006, 27(7) 1454-1464.

15. Stokes, J.E., and Ridgway, G. L. Clinical Bacteriology, F. Dward Arnold, 5.

16. Mackenzie, D.W.R., Philpot, C.M. and Proctor, A.G.J. Basic Serodiagnostic for Disease caused by fungi and Adinomycetes, Public Health Laboratory Science, 1980, 12.

17. Emmons, C.W. Binford; C.H., Utz., J.P. and Knon-Chung, K.J. Medical Mycology, Lea and rebager, Philadelphia, 1977, 3. 